\title{
Poincaré-Birkhoff theorem in quantum mechanics
}

\author{
D. A. Wisniacki, ${ }^{1}$ M. Saraceno, ${ }^{2}$ F. J. Arranz,${ }^{3}$ R. M. Benito, ${ }^{3}$ and F. Borondo ${ }^{4}$ \\ ${ }^{1}$ Departamento de Física and IFIBA, FCEyN, UBA Ciudad Universitaria, Pabellón 1, Ciudad Universitaria, 1428 Buenos Aires, Argentina \\ ${ }^{2}$ Departamento de Física, Comisión Nacional de Energía Atómica, Avenida del Libertador 8250, 1429 Buenos Aires, Argentina \\ ${ }^{3}$ Grupo de Sistemas Complejos and Departamento de Física, Escuela Técnica Superior de Ingenieros Agrónomos, \\ Universidad Politécnica de Madrid, 28040 Madrid, Spain \\ ${ }^{4}$ Departamento de Química, and Instituto Mixto de Ciencias Matemáticas, CSIC-UAM-UC3M-UCM, Universidad Autónoma de Madrid, \\ Cantoblanco, 28049 Madrid, Spain
}

(Received 6 May 2011; revised manuscript received 30 June 2011; published 22 August 2011)

\begin{abstract}
Quantum manifestations of the dynamics around resonant tori in perturbed Hamiltonian systems, dictated by the Poincaré-Birkhoff theorem, are shown to exist. They are embedded in the interactions involving states which differ in a number of quanta equal to the order of the classical resonance. Moreover, the associated classical phase space structures are mimicked in the quasiprobability density functions and their zeros.
\end{abstract}

DOI: 10.1103/PhysRevE.84.026206

PACS number(s): 05.45.Mt, 03.65.Sq

The quantum manifestations of classical chaos, or quantum chaos, have received much attention in the recent past [1]. Besides random matrix theory, which describes universal properties, Gutzwiller's trace formula describes the dual relationship between coherent sums over periodic orbits with sums over quantized states [2]. The appearance of scars of periodic orbits in individual eigenfunctions and the associated scar theory are results that deserve special mention [3-5]. These results are by now routinely applied in a variety of important technological applications [6]. At the other extreme, for integrable systems, this dual relationship is more precise and collective sums over the degenerate periodic orbits on rational tori give rise to individual states on quantized tori [7].

For systems close to integrable, the classical behavior is completely understood in terms of the celebrated KolmogorovArnold-Moser (KAM) and the Poincaré-Birkhoff (PB) theorems $[8,9]$. The first one deals with quasiperiodic motion and the persistence of sufficiently irrational tori under perturbations. The second considers the fate of the (unperturbed) resonant tori, from which an even number of periodic orbits (POs) survive. In their vicinity, chains of islands of regularity surrounded by a chaotic separatrix organized by a homoclinic tangle are formed. This structure and the associated transport are well described by the universal pendulum model of Chirikov [10], where the (slow) Arnold diffusion [8] is the controlling mechanism of transport. Classical resonances are crucial in the control of many relevant processes, such as intramolecular vibrational relaxation in chemistry or directional laser emission in nano-optics. Illustrative examples of this can be found in Refs. [11] and [12], respectively. On the other hand, the analysis of the quantum counterpart has produced many fewer results [13-15].

In this paper, we explore the quantum implications of the classical PB theorem. We find that they exist and can be unveiled by studying the mechanism by which two quantized tori of the unperturbed system interact to form the beginning of an island chain on a resonant rational torus. The subtle mechanism by which the quantum numbers of the unperturbed tori are exchanged in a quasicrossing is illustrated by the exchange of zeros of the stellar representation [16]. The splitting of a series of avoided crossings (ACs) ruled by a $\mathrm{PB}$ resonance in the correlation diagram is explained semiclassically.

The model that we have chosen to study is the Harper map in the unit square,

$$
\begin{aligned}
& p_{n+1}=p_{n}+k \sin \left(2 \pi q_{n}\right) \quad(\bmod 1), \\
& q_{n+1}=q_{n}-k \sin \left(2 \pi p_{n+1}\right) \quad(\bmod 1),
\end{aligned}
$$

where $k$ is a parameter measuring the strength of the perturbation. The quantized version is provided by the unitary time-evolution operator $[17,18]$

$$
\hat{U}=\exp [i N k \cos (2 \pi \hat{q})] \exp [i N k \cos (2 \pi \hat{p})],
$$

with $N=(2 \pi \hbar)^{-1}$. The simplicity of this model allows extremely detailed calculations.

The classical dynamics of this map follows the KAM and PB pattern as a function of $k$, as depicted in Figs. 1(a)-1(d). For small values $k=0.1$ [Fig. 1(a)] all trajectories look regular at the scale of the figure, being confined to invariant tori. For $k=$ 0.155 [Fig. 1(b)] two resonances of order 1:14 and 1:10 around the center become important and the corresponding chains of islands are visible. Also, the separatrix between central tori and those at corners shows as an appreciable band of stochasticity. As $k$ reaches 0.2 [Fig. 1(c)] the classical phase space continues evolving; the 1:14 resonance disappears, swallowed by chaos in the main separatrix, while the 1:10 resonance grows in size, and a new 1:6 resonance becomes apparent. Finally, for $k=0.25$ [Fig. 1(d)] only the 1:6 resonance survives.

The quantum map can be diagonalized, resulting in a set of $N$ eigenphases $\phi_{n} \in[-\pi, \pi)$ and eigenfunctions $\left|\phi_{n}\right\rangle$. Plotted as a function of $k$, the eigenphases produce the correlation diagram in Fig. 1(e). To interpret this diagram we rewrite the map using the Baker-Hausdorff expansion for noncommuting exponentials [19,20] as

$$
\hat{U}=\exp \left[i N k \hat{H}^{(n)}(k)\right] \exp \left[i N V^{(n)}(k)\right],
$$

where $\hat{H}^{(n)}=\hat{H}_{0}+k \hat{H}_{1}+\cdots+k^{n-1} \hat{H}_{n-1}$ and $\hat{V}^{(n)}(k)$ is another operator with a power series expansion starting with the power $k^{n+1}$. All terms of both expansions can be computed in terms of iterated commutators of $\cos (2 \pi \hat{q})$ and $\cos (2 \pi \hat{p})$. If commutators are replaced by Poisson brackets in the usual 


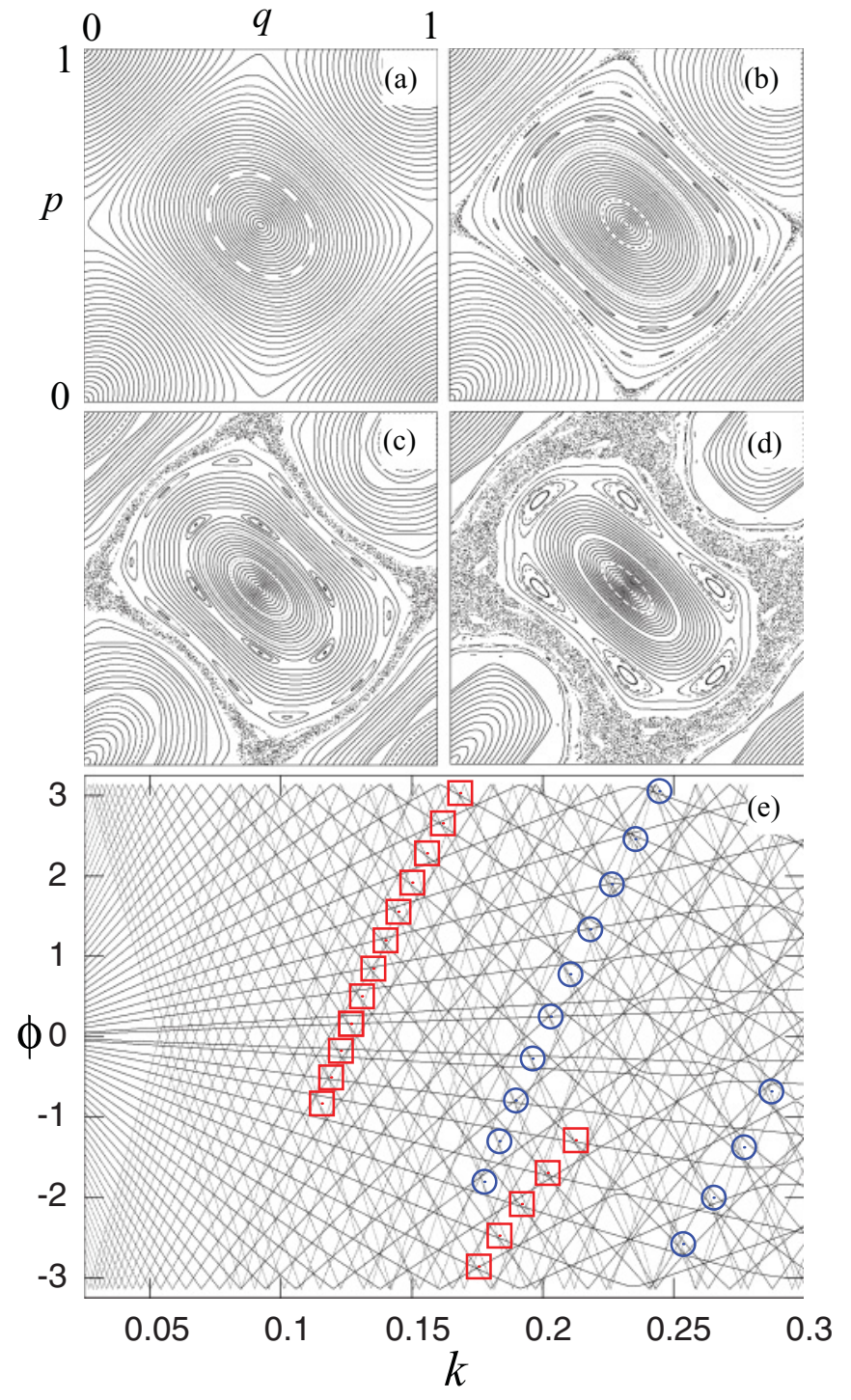

FIG. 1. (Color online) Classical phase space for the Harper map (1) for $k$ (a) 0.1 , (b) 0.155 , (c) 0.2 , and (d) 0.25. (e) Correlation diagram for the eigenphases of the quantized Harper map (2) with $N=60$ as a function of the perturbation parameter $k$. Two series of avoided crossings of states with quantum numbers differing by ten (red squares) and six (blue circles) units, respectively, are shown.

fashion, the equivalent classical expansion is obtained [20]. As an example the classical expansion to second order yields

$$
\begin{gathered}
\hat{H}^{(2)}(p, q)=\frac{\cos (2 \pi \hat{q})+\cos (2 \pi \hat{p})}{2 \pi}+\frac{k}{2} \sin (2 \pi \hat{q}) \sin (2 \pi \hat{p}), \\
\hat{V}^{(2)}(p, q)=-\frac{k^{3}}{12}\left[\cos (2 \pi \hat{q}) \sin ^{2}(2 \pi \hat{p})\right. \\
\left.+\cos (2 \pi \hat{p}) \sin ^{2}(2 \pi \hat{q})\right] .
\end{gathered}
$$

Further terms can be (laboriously) calculated. The importance of this rewriting for our purposes is that for moderate values of $k$ the exact eigenphases can be interpreted as the levels of the integrable operator $\hat{H}^{n}(k)$ with definite quantum numbers $n$. When multiplied by $k N$ and wrapped around the unit circle, the wrapping produces ACs of states with different quantum number which become ACs when the small terms of order $k^{n+1}$ in $\hat{V}$ are taken into account. This procedure eventually becomes divergent, reflecting the fact that the chaotic behavior of the map cannot be captured by any integrable approximation. However, the procedure is well suited to describing the beginning of the PB process when a rational torus disintegrates in an island chain. This situation is clearly visible in the correlation diagram of the eigenphases, calculated for $N=60$. There are 60 eigenlines all starting at zero for $k=0$, and then they spread in a fanlike fashion, first linearly with $k$. The slopes are given initially by the eigenvalues of $\hat{H}_{0}=[\cos (2 \pi \hat{q})+\cos (2 \pi \hat{p})] / 2 \pi$ and they are ordered in increasing value of their quantum number. Half of these lines have positive slope corresponding to states localized around $(p, q)=(0,0)$ while the ones with negative slopes are centered on $(p, q)=(1 / 2,1 / 2)$. We will restrict our analysis to the island formation in this latter case, the other being equivalent since it is connected by a simple symmetry operation.

Two interesting features arise from the analysis of the correlation diagram. First, the ACs always appear grouped in series or families, where the involved states differ by the same number of quanta, $\Delta n$. Two such families of ACs, corresponding to $\Delta n=10$ and $\Delta n=6$, have been identified and marked with red squares and blue circles, respectively, in the correlation diagram of Fig. 1. The detailed analysis of one of these ACs is shown in Fig. 2. The AC involves states with $n_{\text {lower }}=4$ and $n_{\text {upper }}=10$ in the $\Delta n=6$ family and is a typical sharp two-level AC. The left panel of Fig. 2 is a blow-up of Fig. 1 in the range $k=0.185-0.21$. Pairs of points (a),(f), (b), (g), (c),(h), (d),(i), and (e),(j) show different stages of the $\mathrm{AC}$, the minimum $\Delta \phi$ being attained at point (d),(i).

More interesting is the evolution of the associated Husimi functions, which is shown, using the same labeling, in the right panels of the figure. Recall that the Husimi distribution of a quantized torus is localized on it, and its quantum number is given by the number of zeros enclosed by the torus $[16,17]$. The rest of the zeros, in this case to a total of $N=60$, are scattered in the complementary phase space region. Far from the AC, at points [(a),(f) and (b), (g)] the two tori maintain their individuality, but the zeros start moving in a very characteristic way: the lower state $n=4$ attracts six zeros while six zeros start migrating outward in the upper $n=10$ state. The process culminates in panels (d) and (i) where the two states are now precisely localized on the rational torus but with maxima on either the stable or the unstable periodic points given by the PB theorem (the upper state sits on top of the unstable points and the lower on the stable points). Similarly, the positions of the zeros alternate between the fixed points, localizing in the immediate neighborhoods. Beyond the $\mathrm{AC}$, the original topology is restored, aside from the compulsory exchange of quantum numbers. We have confirmed this mechanism for all other ACs of this family and also for the 1:10 resonance. We can then conclude that this is a general mechanism by which two quantized tori interact via periodic orbits - stable and unstable - that, according to the PB theorem, remain on the resonant torus. Even more interesting is the fact that the number of zeros exchanged between the tori is precisely the order of the resonance.

Let us now make the above arguments quantitative. At an $\mathrm{AC}$ the splitting of the levels is given by the interaction 


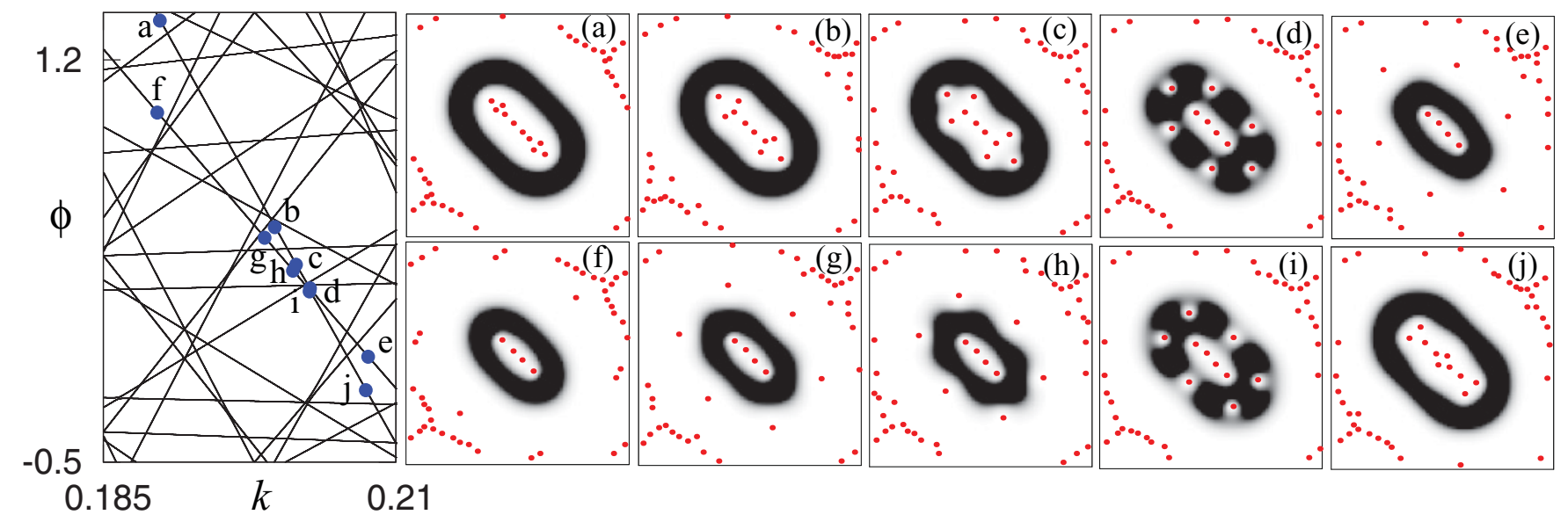

FIG. 2. (Color online) Left: Detail of the correlation diagram of Fig. 1 corresponding to the fifth element of the family of avoided crossings with $\Delta n=6$ which involves states with quantum numbers $n_{1}=4$ and $n_{2}=10$. Right: Evolution of the corresponding quasiprobability densities given by the Husimi functions at selected points $[(\mathrm{a})-(\mathrm{j})]$ in the diagram. Full circles mark the positions of the zeros of the Husimi functions.

matrix element between the two involved states. Quantum mechanically it could be calculated from the eigenstates of the integrable Hamiltonian $H^{(n)}$ perturbed by $V^{(n)}$ in Eq. (3) and computed up to a given power of $k$. However, we are interested in a purely semiclassical calculation. To avoid the very cumbersome transformation to action-angle variables, in which the calculation would be trivial, we resort to a method developed in Ref. [21] in connection with the calculation of tunneling rates in this same model. The resulting expression for the eigenphase difference at the $\mathrm{AC}$ due to a $1: r$ resonance is then obtained in terms of purely classical information as

$$
\hbar \Delta \phi=\frac{1}{8 \pi^{4} r} \int_{0}^{2 \pi} \exp (-i r \theta) \delta I_{1: r}(\theta) d \theta,
$$

$\delta I_{1: r}(\theta)$ being given by

$$
\delta I_{1: r}(\theta)=I^{(-1)}\left(I_{1: r}, \theta\right)-I_{1: r},
$$

where $I^{(-1)}\left(I_{1: r}, \theta\right)$ symbolizes the action variable that is obtained by applying the inverse Poincaré map to $(I, \theta)$ or, alternatively, the backward propagation with $H$ from time $t=\tau$ to $t=0$. The way the calculations are done in practice is the following. First, the resonant periodic torus is located and its action computed. This is done by using an analytical thirdorder approximation to the Harper Hamiltonian in Eq. (3), followed by numerical refinement propagating trajectories near the torus. Once the resonant torus is found, the corresponding angle variable is computed for a large sample of points, which are then back-propagated with the exact inverse map. (This is a trivial process since it just implies exchanging the $p$ and $q$ variables.) The associated action corresponding to each of these points is then calculated by numerical propagation of the trajectories in a complete cycle. This provides the values of the perturbed action, from which $\Delta \phi$ is evaluated with the aid of Eq. (5). The corresponding results for the 1:6 resonance are shown as a continuous line in Fig. 3. As can be seen, the agreement of this semiclassical calculation with the data (triangles) extracted from the correlation diagram of Fig. 1 is extremely good, thus giving full support to our previous qualitative arguments on the existence of a clear quantum manifestation of the classical PB theorem.

A final point worth addressing is the limit $N \rightarrow \infty$ of our results, which is closely related to the extent to which quantum mechanics can distinguish the $\mathrm{PB}$ structure of a given classical resonance. According to the KAM and PB theorems, such structures exist even for infinitesimal values of the perturbation, although as $k$ decreases they span smaller regions of phase space. On the other hand, the finiteness of $\hbar$ imposes a limit to the phase space details which can be resolved quantum mechanically. How the two extremes can be reconciled with our previous conclusions is not clear in principle. For example, in the correlation diagram of Fig. 1 the 1:6 resonance is only observed for $k>0.166$, point at which the first element, i.e., $n_{\text {lower }}=0$, of the corresponding family of ACs is located. Could this resonance be resolved for smaller perturbations if a smaller value of $\hbar$ was chosen? Fortunately, this question can be easily answered in our calculations, where $\hbar$ is a parameter directly related to the size of the Hilbert space, $N=(2 \pi \hbar)^{-1}$. Indeed, the answer to the question is yes, as

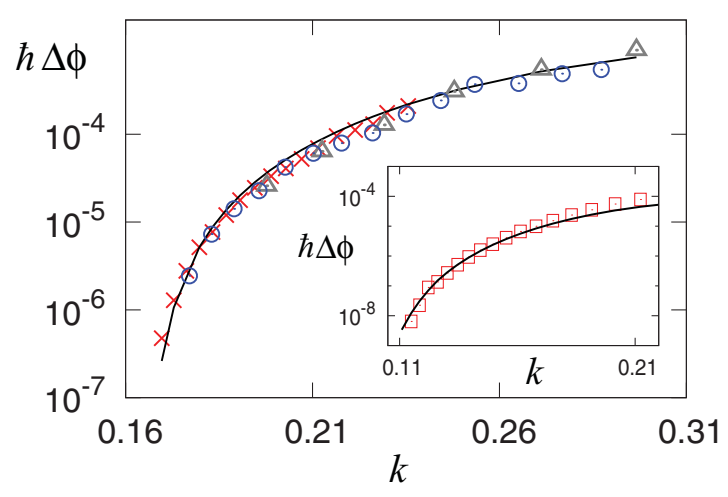

FIG. 3. (Color online) Numerical eigenphase differences $\Delta \phi$ for avoided crossings of the $\Delta n=6$ family extracted from the correlation diagram of Fig. 1 (blue circles) as a function of the perturbation parameter for $N$ : gray $\triangle, 30$; blue $\odot, 60$; red $\times, 100$; and full line, semiclassical calculations based on Eq. (3) for the 1:6 resonance. Inset: Same for the 1:10 resonance and $N=100$. 
demonstrated by Fig. 3, where the results for $\Delta \phi$ for three different values of $N$ are shown. For increasingly larger values of $N$ the first member of the AC series appears at smaller values of $k$, the interaction being satisfactorily described in all cases by the semiclassical theory.

Finally, let us remark that our conclusions are also valid for other resonances and systems. This is illustrated in the inset to Fig. 3, where the results for the resonance 1:10 are shown, and a perfect agreement between quantum and semiclassical results is also seen. Moreover, a similar study has been carried out in a realistic model for the vibrational dynamics of the LiCN molecule [22], arriving at the same conclusions; the results will be reported elsewhere.

Summarizing, in this paper we have shown that quantum manifestations of the PB theorem, a cornerstone to rationalization of classical Hamiltonian chaos, exist. They can be unveiled by analyzing the interactions or AC splittings in a correlation diagram, involving pairs of states which differ in a number of quanta equal to the order of a given classical resonance. This result has remarkable effects, since as a (perturbational) parameter varies and a quantized torus passes through the resonance it loses (or gains) zeros in its Husimi representation. At resonance the zeros are located at the position of the periodic points predicted by the PB theorem, and the number of zeros, lost or gained, is equal to the order of the resonance. Our conclusions are supported with semiclassical calculations of the relevant AC splittings ruled by the PB resonance.

This work was supported by the MICINN-Spain under Contracts iMath-CONSOLIDER No. CDS2006-32 and No. MTM2009-14621, UBACyT ( Grant No. X237), and CONICET (Argentina).
[1] H. J. Stöckman, Quantum Chaos: An Introduction (Cambridge University, Cambridge, UK, 1999).

[2] P. Cvitanović, R. Artuso, R. Mainieri, G. Tanner, and G. Vattay, Chaos: Classical and Quantum (ChaosBook.org, Niels Bohr Institute, Copenhagen, 2009).

[3] Chaos and Quantum Physics, edited by M.-J. Giannoni, A. Voros, and J. Zinn-Justin, Proceedings of the Les Houches Summer School of Theoretical Physics, 1989 (North-Holland, Amsterdam, 1991).

[4] E. J. Heller, Phys. Rev. Lett. 53, 1515 (1984).

[5] D. A. Wisniacki, E. Vergini, R. M. Benito, and F. Borondo, Phys. Rev. Lett. 94, 054101 (2005); 97, 094101 (2006).

[6] P. B. Wilkinson et al., Nature (London) 380, 608 (1996); J. U. Nockel and A. D. Stone, ibid. 385, 45 (1997); C. Gmachl et al., Science 280, 1556 (1998); C. Michel, V. Doya, O. Legrand, and F. Mortessagne, Phys. Rev. Lett. 99, 224101 (2007).

[7] M. V. Berry and M. Tabor, Proc. R. Soc. London Ser. A 349, 101 (1976).

[8] V. I. Arnold, S. M. Gusein-Sade, and A. N. Varchenko, Mathematical Aspects of Classical and Celestial Mechanics (Springer-Verlag, Berlin, 2006).

[9] G. D. Birkhoff, Trans. Am. Math. Soc. 14, 14 (1913).
[10] B. V. Chirikov, Phys. Rep. 52, 263 (1979).

[11] S. Keshavamurthy, Int. Rev. Phys. Chem. 26, 521 (2007).

[12] J. Wiersig, Phys. Rev. Lett. 97, 253901 (2006); J. Unterhinninghofen, J. Wiersig, and M. Hentschel, Phys. Rev. E 78, 016201 (2008).

[13] G. Hose and H. S. Taylor, Phys. Rev. Lett. 51, 947 (1983).

[14] T. Geisel, G. Radons, and J. Rubner, Phys. Rev. Lett. 57, 2883 (1986).

[15] D. M. Leitner and P. G. Wolynes, Phys. Rev. Lett. 79, 55 (1997); M. Gruebele and P. G. Wolynes, ibid. 99, 060201 (2007).

[16] P. Leboeuf and A. Voros, J. Phys. A 23, 1765 (1990).

[17] P. Leboeuf, J. Kurchan, M. Feingold, and D. P. Arovas, Phys. Rev. Lett. 65, 3076 (1990).

[18] P. Bianucci, J. P. Paz, and M. Saraceno, Phys. Rev. E 65, 046226 (2002).

[19] H. F. Baker, Proc. London Math. Soc. 34, 347 (1902).

[20] R. Scharf, J. Phys. A 21, 2007 (1988).

[21] O. Brodier, P. Schlagheck, and D. Ullmo, Phys. Rev. Lett. 87, 064101 (2001); Ann. Phys. 300, 88 (2002).

[22] F. J. Arranz, R. M. Benito, and F. Borondo, J. Chem. Phys. 123, 044301 (2005). 